\title{
Current Challenges and Opportunities for Space Technologies
}

\author{
Guglielmo S. Aglietti* \\ Department of Mechanical Engineering, The University of Auckland, Auckland, New Zealand
}

Keywords: space technologies, satellite technologies, space propulsion, space debris, launch vehicles, spacecraft structures, large space structures (LSSs), active debris removal (ADR)

\section{SETTING THE SCENE}

With the launch of Sputnik in 1957 and the subsequent beginning of the space age, the progression of Space Technologies has, on the one hand, led to the development of hundreds of applications (Pelton et al., 2017) that use satellite data, including devices for everyday use, from satellite televisions to the Satnav in our cars. On the other, it has underpinned scientific progress in Earth and Atmospheric Sciences as well as in Astronomy and Astrophysics. Just to recall some of the highest public profile contributions from the field, satellite measurements showed the extent of the ozone layer depletion in the atmosphere and the existence of exoplanets and black holes have been confirmed, among many other scientific advances.

The rapid progress made in Space Technology led to extraordinary accomplishments for the whole human race, such as the Moon landing.

At the same time, these space missions have provided powerful iconic imagery for humanity, and photos like the Blue Marble (Wuebbles, 2012) have become universally recognized symbols of our planet and its extraordinary environment and finite resources.

Although the spectacular progress in Space Technologies slowed down toward the end of the past century, together with that of the whole Aerospace sector, very important achievements continued to be made. These include the development of the International Space Station and the robotic exploration of other planets and celestial bodies, including landing on a comet!

Through the years, space has often been identified as the new frontier, fueling the imagination

Edited and reviewed by: Jack J. W. A. van Loon Vrije Universiteit Amsterdam, Netherlands

*Correspondence: Guglielmo S. Aglietti g.aglietti@auckland.ac.nz

Received: 04 May 2020 Accepted: 13 May 2020 Published: 16 June 2020

Citation:

Aglietti GS (2020) Current Challenges and Opportunities for Space Technologies.

Front. Space Technol. 1:1. doi: 10.3389/frspt.2020.00001 of writers and film directors, who created visions (more or less plausible) of a future enabled by fantastic developments in Space Technologies.

However, consistent with what history has shown us, is the fact that, after an initial phase of "exploration" of a new environment and consolidation of the relevant technologies, what follows is an explosion of businesses to exploit the new opportunities offered by the new environment. This is where we are today. Sometimes called Space 4.0, we are in a period that has seen a shift of paradigms, with changes of motivations, actors, and, indeed, technologies (PWC Report, 2019).

\section{NEW SPACE AND THE NEED FOR APPROPRIATE REGULATORY FRAMEWORK}

Under the name of "New Space", there is an ongoing "revolution" in the space sector with new players/commercial entrepreneurs/businesses (Hall, 2020) entering a domain traditionally occupied by institutional players ("Old Space” e.g., space agencies working with large companies) to

\footnotetext{
${ }^{1}$ https://en.wikipedia.org/wiki/NewSpace (accessed March 4, 2020)
} 
exploit the new opportunities opening in front of them. These could include new services, offered through applications of space data (from precision navigation/agriculture, surveillance, to Earth environment monitoring, etc.), to more futuristic opportunities, such as space tourism or asteroid mining. Successful entrepreneurs from other sectors, from Richard Branson in the UK to Elon Musk in the US, have entered the "space" arena, taking risks and challenging the conservativism of the established "Old Space" business model.

Here is where we can identify a first challenge and learn a lesson from history, preventing space from becoming a lawless "wild west" where the strongest can take an unfair advantage. This should apply to Low Earth Orbit (LEO), where the current regulatory framework should be further developed and enforced to manage the increasing "space traffic" (Cukurtepe and Akgun, 2009; Lal et al., 2018) (to prevent interferences or collisions between assets of different operators), as well as to Medium (MEO) or Geosynchronous Earth Orbit (GEO) and interplanetary exploration and exploitation. Indeed, new regulations should be implemented respecting established treaties and principles (e.g., "Outer Space Treaty" or the "Convention on International Liability for Damage Caused by Space Objects").

In areas where an appropriate regulatory framework is already in operation and largely followed by all the stakeholders, like the satellite telecommunication sector (ITU, 2012), the challenge becomes to stay abreast with technology progress and market evolution (Morozova and Vasyanin, 2019). Together with the challenge, there is an opportunity to develop new regulations that spur further technological progress.

The issue of the overcrowded radio-frequency spectrum, as well as the sustainability of the space environment which is threatened by the growing amount of space debris, have to be tackled. Solutions that are acceptable to the various stakeholders (from commercial organization to political entities) have to be found and, most importantly, implemented.

Activities in space cannot be contained within the boundary of a country's border and have the potential to affect assets or areas of the planet well-beyond the jurisdiction of a launching country or the nation where a satellite operator is registered. Therefore, the international regulatory framework should clearly prevail on national regulations and limit the capability of countries to use less stringent regulations as a means to attract foreign business. At the same time, some consideration also has to be given to how to enforce at an international level the agreed rules and regulations.

Indeed, the development of rules and regulations must be mitigated to avoid unnecessary red tape stifling new enterprises, and space law should preserve the freedom to generate new ideas and implement new applications. Hence the challenge lies in balancing these competing requirements: a regulatory framework which protects stakeholders, countries' interests, and current and future human rights on one side, with the freedom to develop and exploit new technologies on the other.

\section{TECHNICAL CHALLENGES}

\section{Propulsion Systems}

Coming now to the more technical challenges, propulsion systems performance is a significant hurdle to overcome in the space sector (Turner et al., 2009).

Starting with the launch vehicles, their capabilities (broadly speaking, payload size, and thrust) have essentially plateaued, as only relatively minor incremental progress has been made in the last decades. Indeed, materials have improved with the introduction of composites with mechanical properties far superior to the typical alloys that were used at the beginning of the space age. Design and manufacturing techniques have also improved, with progress in software simulation enabled by the extraordinary growth of computer power, or new manufacturing methods such as additive manufacturing. Guidance and control systems have also improved thanks to advances in electronics and software. However, besides the push toward green propellants (Gohardani et al., 2014), nothing substantial has changed with the solid or liquid propellants performance and related technologies, which are key to overall launcher capability (Devezas, 2018). Reusable launchers are being used by a few companies in order to reduce costs or increase launch frequencies, and it is undeniable that costs have slowly come down, although this is largely due to combinations of countries' policies and market forces, but truly economic access to space is yet to be achieved.

On a longer timescale, the challenge is to develop and implement technologies, such as hypersonic air breathing rocket engines $^{2}$, to be used in hybrid launchers to cut the need for large amounts of oxygen that have to be carried by current vehicles. Launch vehicles that could take off and land as aircraft, without the need for extensive and expensive service between missions, should also be developed. Similarly, inspace propulsion offers opportunities for improvement, in particular on Electric propulsion systems, which are also hybrid systems that would utilize different modes of operation (Levchenko et al., 2020).

Propulsion systems performance are also fundamental to interplanetary missions in terms of enabling faster travel and larger payloads to be delivered where required. Our current limitations in the manned exploration of the solar system are mainly due to the length of travel, which is directly related to the level of performance that is available from existing propulsion systems. Similarly, propulsion systems performance limits our exploration and exploitation capabilities (also for robotic activities) as it is a significant limitation to the mass of payload that can be safely transported to/from other celestial bodies.

\section{PROTECTION OF HUMANS}

Strictly related to human exploration are space health and medicine (Hodkinson et al., 2017), to enable humans to withstand the space environment for long periods, and the creation of artificial habitats in space and on other planets

\footnotetext{
${ }^{2}$ https://en.wikipedia.org/wiki/SABRE_(rocket_engine)
} 
to support a reasonable quality of human life. The challenge here consists in the creation of a whole artificial environment to support people's well-being and physical and mental health, with means protecting against the negative effects of the space environment (Grimm, 2019). Whether we are considering a manmade vessel for long distance space travel or a space platform for large-scale human inhabitation or a planetary colony, some challenges overlap. These overlapping challenges include the need to create efficient closed loop systems to replenish resources and minimize waste, with the common goal to generate an artificial ecosystem for the long-term support of human life ${ }^{3}$.

Another aspect of our need to protect and preserve human life is the issue of planetary defense (Simpson, 2015).

Near Earth Objects like asteroids or comets can pose a significant threat. Although smaller objects (such as meteors) hit our planet daily and mostly burn up whilst traveling though the atmosphere, some larger objects can survive and hit the Earth's surface with significant energy. Most of the craters produced by the larger objects that have hit Earth since its formation have been erased by the planets geological processes, but there are dozens of large craters still visible (a great example is the 1 kilometer wide Barringer Meteor Crater in Arizona, which was formed $\sim 50,000$ years ago by the impact of a metallic object $\sim 50 \mathrm{~m}$ in diameter) that serve as reminders that these impacts can occur. Depending on the size of the object, which could be 100's of meters or kilometers in diameter, and the location of the impact, consequences could range from relatively minor damage, to a major catastrophe that could destroy life on vast regions of the planet, if not all life entirely. Fortunately, the probability of these events is very low, but their consequences are dramatic, hence appropriate mitigation strategies must be developed. All major space organizations (NASA, ESA, etc.) have given some attention to this issue (Office of Audits, 2014) [ESA Asteroid Impact Mission (AIM) and NASA Double Asteroid Redirection Test (DART)], and the United Nations have taken good steps to improve coordination, establishing the International Asteroid Warning Network and the Space Mission Planning Advisory Group (SMPAG). However, as this is a global threat, a greater level of international coordination and integration of the efforts made by single entities is necessary to produce an effective response. Should the threat of catastrophic impact from a large asteroid materialize, the world cannot afford a disorderly and fragmented response, such as that seen with past global crises (e.g., the Covid-19 pandemic). Time will be limited so the plans have to be prepared and agreed upon, ready to be executed in order to produce a timely response and perform the necessary mission.

From a technical perspective, besides the challenges to improve detection capabilities and potential impact predictions, the development and testing of methodologies and technologies to deflect a large object (as this currently seems the most realistic and effective method of intervention) have to progress to the point that it could be deployed with a high level of confidence in its success.

$\overline{{ }^{3} \text { https://www.nasa.gov/content/life-support-systems }}$

\section{EARTH ENVIRONMENT}

Following the theme of the protection of the Earth, climate change is a significant threat to our environment with potentially disastrous consequences, and is an area where Satellite Technologies can help in tackling the challenge (Brünner et al., 2018). On a global scale, satellites provide impartial information to monitor the environment and develop and validate models improving our predictive capabilities. But greater effort has to be placed in producing actionable information related to specific needs and issues, reducing the vast amount of data to simpler interpretations that can inform the political discourse.

Both institutional and private Earth Observation (EO) markets demand very high resolution and coverage, as well as a short revisit time, with the aim to achieve real time Earth Observation. From surveillance to disaster monitoring and management of resources, there are literally hundreds of applications, but still there is a relatively low market adoption. This becomes obvious if we compare satellite EO with satellite telecommunications, as in the latter sector there is an abundance of purely commercial organizations that operate (without institutional support) in a user-driven market, whereas the EO market is still most often enabled by institutional budgets. There is a clear trend toward a more commercial EO sector that is facilitated by smaller sized and cheaper satellites (Rycroft and Crosby, 2013), and several companies offering cost effective solutions promising technically-acceptable performance at a price that the market can bear. However, there is very high competition in a market that still has too small a number of customers.

\section{LOW COST SPACE TECHNOLOGIES}

This democratization and marketization of space is evident in the growth of the cubsat market (Davoli et al., 2019). Here, space $\mathrm{HW}$ is available at prices so low that it has attracted a growing number of customers (from Space Agencies to institutions like universities and schools), which in turn have enabled the creation of start-ups and spinoffs. However, the performance of these systems are very limited, and often the constraints come from their physical size (e.g., the size of the optics limits the resolution that can be achieved, or the size of the solar panels limits the amount of power that can be collected). This has led to the development of deployable structures to package relevant elements into small (cubesat compatible) volumes and then deploy them in space to achieve the required level of performance. Sometime these act as demonstrations for applications aimed at larger satellites, like drag sails (Underwood et al., 2019).

There are other technical issues that affect all smaller mass satellites, not just cubesats, and present significant challenges, like the need to achieve high platform stability. This is crucial for all missions supporting highly accurately targeted optical payloads (e.g., high-resolution cameras/telescopes or laser communication systems) or devices that demand stability for inertial types of measurements. The issue lies with the minimization of the microvibrations that are produced by essential on-board equipment 
(Remedia et al., 2015), and that can, for example, produce unacceptable oscillations of the line of sight. This issue is more severe for smaller craft as there is less mass (inertia), which naturally reduces the vibration level. Ground testing and modeling to predict in-orbit performance are still inaccurate and practical applications rely on the use of large margins rather than accurate models. Active control of micro-vibrations is still very difficult and too expensive to be implemented, in particular at the lower end of the market, where most growth is expected.

In terms of their overall performance, cubesat capabilities are often oversold to an inexperienced public, and there is a general need for higher quality products with the involved hardware that still maintain affordability.

\section{LARGE SPACE STRUCTURES}

At the other end of the spectrum, with respect to cubesats, in terms of size, are Large Space Structures (LSS). These have been considered and studied for decades, but real progress has been slow.

The capability to deploy LSS is another factor that, similarly to advances in propulsion, would enable a range of applications, but they present a series of significant challenges which depend on the specific areas.

On the one hand there are instruments like telescopes, cameras, and antennas that require large $(>10 \mathrm{~m}$ and possibly an order of magnitude larger) high-precision reflective surfaces. Here the current methodologies (e.g., based on the deployment of precisely machined and polished mirrors whose positions and shape can be adjusted by a series of actuators) are limited by the size and number of segments that can be deployed [e.g., James Webb Space Telescope $(J W S T)^{4}$ ], and the overall staggering cost. Concerning the antennas, a variety of deployable solutions have been proposed, from inflatables to tensegrity structures, but Europe still needs to develop an appropriate commercial solution for current and future applications. Overall, new lighter-weight technologies have to be deployed, to increase packaging efficiency without compromising the quality of the final reflector.

On the other hand, there are future applications like Satellite Solar Power ${ }^{5}$ where the sheer size of the structure (square kilometers) is the challenge, rather than the geometrical precision that must be achieved by the assembled structure. Extreme light

${ }^{4}$ https://www.jwst.nasa.gov/content/observatory/ote/mirrors/index.html

${ }^{5}$ Solar power satellites DM Flournoy, Springer, 2011 - books.google.com

\section{REFERENCES}

Aglietti, G. S., Taylor, B., Fellowes, S., Salmon, T., Retat, I., Hall, A., et al. (2020). The active space debris removal mission RemoveDebris. Part 2: in orbit operations. Acta Astron. 168, 310-32. doi: 10.1016/j.actaastro.2019.09.001

Brünner, C., Königsberger, G., Mayer, H., and Rinner, A. (eds.) (2018). Satellite-Based Earth Observation Trends and Challenges for Economy and Society. Springer. weight and packaging efficiency have to be achieved, as well as in-orbit deployment and assembly capabilities that are beyond the state of the art.

\section{IN-ORBIT SERVICING AND ACTIVE DEBRIS REMOVAL}

This brings us to the opportunities offered by robotic in-orbit servicing and the development of flexible technologies that can support multipurpose missions. These opportunities include the servicing and potential repairing of current satellites, to active debris removal. These are not new concepts, as in 1984 the Space Shuttle Discovery mission STS-51-A, brought back to Earth two old satellites no longer functioning (probably the first example of Active Debris Removal), and similarly the mission of the shuttle Endeavor in 1993 (and other following missions) provided essential fixes and services to the Hubble Space Telescope. However, the opportunity here is to develop robotic technologies (Wilde et al., 2019) able to perform these type of missions (Forshaw et al., 2016) at a fraction of the cost. The non-cooperative nature of the target, which could be tumbling, presents the first challenge to any approaching vehicle that has to rendezvous with this object. Techniques to stabilize the target and devices to safely grasp it have to be developed (and standardized) as well as improvements made in the relative navigation (vision-based navigation), hardware, and software. Some progress has been made and some devices tested in-orbit (Aglietti et al., 2020), but we are still far from a real capability to perform Active Debris Removal or in-orbit servicing with sufficient confidence and at an affordable price.

This article has described some of the challenges that the space sector is facing, but there are more, and these come with opportunities for new developments (for example, those offered by multifunctional space structures (Sairajan et al., 2016), or technologies like optical and quantum intersatellite communication Liao et al., 2017). The solutions to the various issues, which in time will be developed, will provide stepping stones for future applications and enterprises that will benefit societies across the world.

\section{AUTHOR CONTRIBUTIONS}

The author confirms being the sole contributor of this work and has approved it for publication.

Cukurtepe, H., and Akgun, I. (2009). Towards space traffic management system. Acta Astron. 65, 870-878. doi: 10.1016/j.actaastro.2009.03.063

Davoli, F., Kourogiorgas, C., Marchese, M., Panagopoulos, A., and Patrone, F. (2019). Small satellites and CubeSats: survey of structures, architectures, and protocols. Int. J. Satellite Commun. Netw. 37, 343-359. doi: 10.1002/sat.1277

Devezas, T. C. (2018). Space propulsion: a survey study about current and future technologies. J. Aerosp. Technol. Manage. 10. doi: 10.5028/jatm.v10.829. [Epub ahead of print]. 
Forshaw, J. L., Aglietti, G. S., Navarathinam, N., Kadhem, H., Salmon, T., Pisseloup, A., et al. (2016). RemoveDEBRIS: an in-orbit active debris removal demonstration mission. Acta Astron. 127, 448-463. doi: 10.1016/j.actaastro.2016.06.018

Gohardani, A. S., Stanojev, J., Demairé, A., Anflo, K., Persson, M., Wingborg, N., et al. (2014). Green space propulsion: opportunities and prospects. Prog. Aerosp. Sci. 71, 128-149. doi: 10.1016/j.paerosci.2014.08.001

Grimm, D. (2019). Guest edited collection: gravitational biology and space medicine. Sci. Rep. 9:14399. doi: 10.1038/s41598-019-51231-8

Hall, M. (2020). Space Philosophies: Who, How, What?. Space Daily. Available online at: https://www.spacedaily.com/reports/NewSpace_Philosophies_Who_ How_What_999.html

Hodkinson, P. D., Anderton, R. A., Posselt, B. N., and Fong, K. J. (2017). An overview of space medicine. Br. J. Anaesth. 119, i143-i153. doi: $10.1093 / \mathrm{bja} / \mathrm{aex} 336$

ITU (2012). Regulation of Global Broadband Satellite Communications. Available online at: http://www.itu.int/ITU-D/treg/broadband/ITU-BB- Reports_ RegulationBroadbandSatellite.pdf

Lal, B., Balakrishnan, A., Caldwell, B. M., Buenconsejo, R. S., and Carioscia, S. A. (2018). Global Trends in Space Situational Awareness (SSA) and Space Traffic Management (STM). IDA Document D-9074 Science \& Technology Policy Institute.

Levchenko, I., Xu, S., Mazouffre, S., Lev, D., Pedrini, D., Goebel, D., Garrigues, L., et al. (2020). Perspectives, frontiers, and new horizons for plasmabased space electric propulsion editors-pick. Phys. Plasmas 27:020601. doi: 10.1063/1.5109141

Liao, S., Yong, H., Liu, C., Shentu, G.-L., Li, D-D., Lin, J., et al. (2017). Long-distance free-space quantum key distribution in daylight towards intersatellite communication. Nature Photon 11, 509-513. doi: 10.1038/nphoton. 2017.116

Morozova, E., and Vasyanin, Y. (2019). International Space Law and Satellite Telecommunications. Oxford Research Encyclopedia of Planetary Science. Available online at: https://oxfordre.com/planetaryscience/view/10.1093/ acrefore/9780190647926.001.0001/acrefore-9780190647926-e-75

Office of Audits (2014). NASA's Efforts to Identify Near-Earth Objects and Mitigate Hazards IG-14-030 (A-13-016-00). Office of Audits - Audit Report.

Pelton, J. N., Madry, S., and Camacho-Lara, S. (2017). Handbook of Satellite Applications. Springer.
PWC Report (2019). Main Trends and Challenges in the Space Sector. Available online at: https://www.pwc.fr/fr/assets/files/pdf/2019/06/fr-pwc-main-trendsand-challenges-in- $\sim$ the-space-sector.pdf

Remedia, M., Aglietti, G. S., and Richardson, G. (2015) A stochastic Methodology for predictions of the environment created by multiple microvibration sources. J. Sound Vibr. 344, 138-157. doi: 10.1016/j.jsv.2015.01.035

Rycroft, M. J., and Crosby, N. (2013). Smaller Satellites: Bigger Business?: Concepts, Applications and Markets for Micro/Nanosatellites in a New Information World. Springer.

Sairajan, K. K., Aglietti, G. S., and Mani, K. M. (2016). A review of multifunctional structure technology for aerospace applications. Acta Astron. 120, 30-42. doi: 10.1016/j.actaastro.2015.11.024

Simpson, M. K. (2015). "Planetary defense, global cooperation and world peace," in Handbook of Cosmic Hazards and Planetary Defense, eds J. Pelton and F. Allahdadi (Cham: Springer), 1055-1067.

Turner, M. J. L., Salgado, M. C. V., and Belderrain, M. C. N. (2009). Rocket and Spacecraft Propulsion: Principles, Practice and New Developments, 3rd Edn. Springer; Praxis Books.

Underwood, C., Denis, A., Viquerat, A., Taylor, B., Sanders, B., Stewart, B., et al. (2019). Inflatesail de-orbit flight demonstration results and follow-on drag-sail applications. Acta Astronautica 162, 344-358. doi: $10.1016 /$ j.actaastro.2019.05.054

Wilde, M., Harder, J., and Stoll, E. (2019). On-orbit servicing and active debris removal: enabling a paradigm shift in spaceflight. Front. Robot. AI. 6:136. doi: 10.3389/frobt.2019.00136

Wuebbles, D. J. (2012). Celebrating the "Blue Marble". EOS Trans. Am. Geophys. Union 93, 509-510. doi: 10.1029/2012EO490001

Conflict of Interest: The author declares that the research was conducted in the absence of any commercial or financial relationships that could be construed as a potential conflict of interest.

Copyright $\odot 2020$ Aglietti. This is an open-access article distributed under the terms of the Creative Commons Attribution License (CC BY). The use, distribution or reproduction in other forums is permitted, provided the original author(s) and the copyright owner(s) are credited and that the original publication in this journal is cited, in accordance with accepted academic practice. No use, distribution or reproduction is permitted which does not comply with these terms. 